Western University Scholarship@Western

Centre for Decision Sciences and Econometrics

Technical Reports

Economics Working Papers Archive

1985

\title{
Auctions with a Stochastic Number of Bidders
}

R. Preston McAfee

John McMillan

Follow this and additional works at: https://ir.lib.uwo.ca/economicscdse_tr Part of the Economics Commons

Citation of this paper:

McAfee, R. Preston, John McMillan. "Auctions with a Stochastic Number of Bidders." Centre for Decision Sciences and Econometrics Technical Reports, 9. London, ON: Department of Economics, University of Western Ontario (1985). 


\title{
THE CENTRE FOR DECISION SCIENCES AND ECONOMETRICS
}

\author{
Auctions with a Stochastic Number of Bidders
}

R. Preston McAfee and John McMillan

\section{TECHNICAL REPORT NO. 9}

DECEMBER 1985

$\dddot{3}$

Centre For Decision Sciences And Econometrics Social Science Centre

The University of Western Ontario London, Ontario N6A 5C2

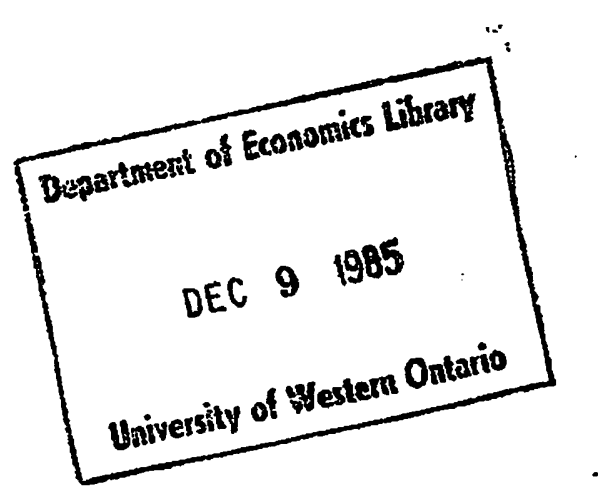




\title{
AUCTIONS WITH A STOCHASTIC NUMBER OF BIDDERS*
}

\author{
R. Preston McAfee and John McMillan
}

Revised: November 1985

\begin{abstract}
Auction theory is generalized by allowing the number of bidders to be stochastic. In a first-price sealed-bid auction with bidders having constant absolute risk aversion, the expected selling price is higher when the bidders do not know how many other bidders there are than when they do know this. Thus the seller should conceal the number of bidders if he can. Moreover, a bidder's ex ante expected utility is the same whether or not there is a policy of concealing the number of bidders: concealment therefore Pareto-dominates announcement. With risk-neutral bidders, the optimal auction is the same whether or not the bidders know who their competitors are.
\end{abstract}

Correspondence to: R. P. McAfee Department of Economics University of Western Ontario London, Ontario, N6A 5C2 Canada

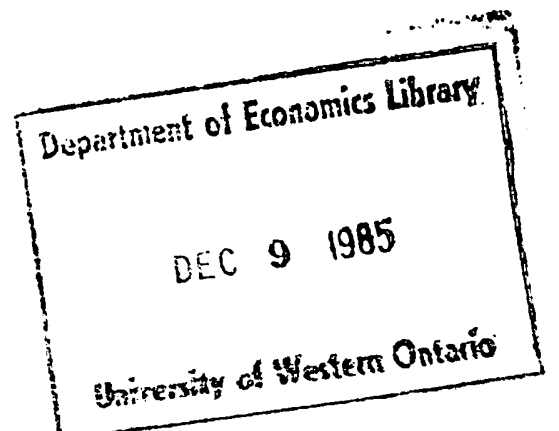




\section{Introduction}

Asymmetric information and imperfect competition are the two essential ingredients of the theory of auctions. ${ }^{1}$ But it is presumed in the existing auction models that one piece of information is common knowledge: all bidders know how much competition they face. Is it appropriate to model the bidders as knowing who their competitors are?

In an English auction, a bidder often cannot identify his rivals. The other bidders may be acting on behalf of anonymous principals. Not all the people present are active bidders. Bidders use subtle signals to hide their bidding. "Such signals may be in the form of a wink, a nod, scratching an ear, lifting a pencil, tugging at the coat of the auctioneer, or even staring into the auctioneer's eyes - all of them perfectly legal. This method of communicating bids gives the process of bidding an aura of secrecy." (Cassady $[1$, pp. 149-150]).

In a sealed-bid auction, there is still less reason to suppose that bidders know the number or the identities of their competitors, since the bidders do not assemble together in one place. With some government-contract bidding, the government invites selected contractors to submit bids. In this case, the government knows in advance the number of bidders. The government therefore has available an extra policy instrument for fostering competition among the bidders, in addition to choosing the form of the auction, setting reserve prices, etc: the government can choose either to conceal or to reveal the number of bidders. ${ }^{2}$

Are the results of auction theory sensitive to the assumption that each bidder knows exactly how many bidders there are? This paper will show that they are. First, if the bidders are risk averse (with constant absolute risk 
aversion) the seller's expected revenue in a first-price sealed-bid auction is higher if the bidders do not know how many bidders there are than if they do know this. Second, with risk-neutral bidders, the optimal direct, incentive-compatible auction is the same whether or not the bidders know the number and the identities of the other bidders. However, knowledge about the set of bidders still matters, because if different bidders have different (albeit Bayesian consistent) expectations over the set of bidders, then if the set of bidders is not known, the optimal auction cannot be implemented using a first-price sealed-bid auction, although it can be implemented using an English auction.

Milgrom and Weber [12] showed that, in many circumstances, it is in the seller's interest to reveal any information he has. This paper exhibits a different set of circumstances in which the seller should conceal information. ${ }^{3}$

In Section 2, we examine the effects of the release of any affiliated information by the seller in a first-price sealed-bid auction with bidders who are risk-averse (having constant absolute risk aversion) and have private values. In the independent-private-values case, we prove that the seller earns more revenue on average when information is concealed than when information is revealed. Interestingly, the bidders are on average equally well off under either policy. In particular, these results apply to information about the number of bidders. Thus, if the seller can choose between concealing and revealing the number of bidders, the policy of concealment is better in the Pareto sense than the policy of revelation. In Section 3, we allow the bidders to be different ex ante, in the sense that their valuations of the good are drawn from different distributions. In addition, they may have different priors on how many bidders are present as 
long as these priors are Bayesian consistent. Thus, revealing the number of bidders is not the only issue, for the seller could also reveal the identities of the bidders, as they are not ex ante the same. If the bidders are risk neutral, the seller is indifferent between revealing and concealing the bidders' identities; his expected revenue is the same in either case. To prove this, we construct the optimal auction, extending the optimal-auctions literature to the case of unknown bidders.

\section{Information Release}

In this section we consider a first-price sealed-bid auction; no reserve price is imposed by the seller. The bidders have symmetric private values, drawn from a density $f$. Assume $f$ is continuous and $f(x, s)$ is strictly positive if $x \in \underset{i=1}{\mathrm{~m}}(\underline{x}, \bar{x})$. Here $x$ is an m-tuple of values, with $m$ the number of potential bidders, and s represents information which may be available to the seller. Initially, we do not require the values to be drawn independently: thus our model is more general than the independent-private-values model, but more special than the general symmetric model of Milgrom and Weber [12].

Following Matthews [4] and Milgrom and Weber [12], we assume the bidders have the same constant-absolute--risk-aversion utility function,

$$
u(w)=\frac{1-e^{-\lambda w}}{\lambda},
$$

for some constant $\lambda \geq 0$.

Using the notation of Milgrom and Weber [12], represent by $x_{1}$ the value of agent 1 and by $Y=\max \left\{x_{2}, x_{3}, \ldots\right\}$ the first (highest) order statistic of the other agent's values. Let

$$
F_{Y}(y \mid x)=\operatorname{Prob}\left(Y \leq y \mid X_{1}=x\right) \text {, }
$$




$$
f_{Y}(y \mid x)=\frac{\partial}{\partial y} F_{Y}(y \mid x) .
$$

Note that

$$
\left.\frac{\frac{\partial}{\partial x} F_{Y}(y \mid x)}{F_{Y}(y \mid x)}\right|_{y=X}=\frac{d}{d x} \log F_{Y}(x \mid x)-\frac{f_{Y}(x \mid x)}{F_{Y}(x \mid x)} .
$$

Consider a symmetric Bayes-Nash equilibrium. Suppose all the bidders other than bidder 1 use a bidding function $B\left(x_{i}\right)$. The function $B$ can be assumed to be increasing, since no agent will ever choose to bid in a nonincreasing portion (simply because, in a nonincreasing portion, it would be in the bidder's interest to reduce his bid, as by doing so he pays less and obtains the good with a higher probability). Thus bidder I's expected utility, if he bids $b$ and has value $x$, is

$$
U(b, x)=\frac{1}{\lambda}\left[1-e^{-\lambda(x-b)}\right] F_{Y}\left(B^{-1}(b) \mid x\right) .
$$

If $B$ is a Nash strategy, then applying the Envelope Theorem to (5) yields

$$
\begin{aligned}
\frac{d}{d x} U(B(x), x) & =\left.\frac{\partial}{\partial x} U(b, x)\right|_{b=B(x)} \\
& =e^{-\lambda(x-B(x))} F_{Y}(x \mid x)+\left.\frac{1}{\lambda}\left[1-e^{-\lambda(x-B(x))}\right] \frac{\partial}{\partial x} F_{Y}(B-1(b) \mid x)\right|_{b=B(x)} \\
& =F F_{Y}(x \mid x)+\left[\frac{d}{d x} \log _{Y}(x \mid x)-\frac{f_{Y}(x \mid x)}{F_{Y}(x \mid x)}-\lambda\right] U(B(x), x)
\end{aligned}
$$

[the last step using (4) and (5)]. Direct integration, using $B(0)=0$ and $F_{Y}(0 \mid 0)=0$, yields that the Nash bidding function B must satisfy: ${ }^{4}$ 


$$
U(B(x), x)=F_{Y}(x \mid x) \int_{0}^{x} \exp \left[-\int_{\alpha}^{x}\left[\frac{f_{Y}(v / v)}{F_{Y}(v \mid v)}+\lambda\right] d v\right] d \alpha .
$$

We say that information is affiliated if the vector of values $x$ together with the information variables satisfies the definition of affiliation given by Milgrom and Weber [12, p. 1098].

THEOREM 1. The public revelation of affiliated information in a first-price sealed-bid auction cannot increase any bidder's utility.

Proof. From (5), a bidder's expected utjlity conditional on winning is

$$
\begin{aligned}
\Pi(x) & =\frac{1}{\lambda}[1-\exp \{-\lambda(x-B(x)]] \\
& =\int_{0}^{x} \exp \left\{-\int_{\alpha}^{x}\left[\frac{f_{Y}(v, v)}{F_{Y}(v, v)}+\lambda\right] d v\right\} d \alpha .
\end{aligned}
$$

Thus

$$
\Pi^{\prime}(x)=1-\left[\lambda+\frac{f_{Y}(x \mid x)}{F_{Y}(x \mid x)}\right] \Pi(x) .
$$

Define a ficitious bidding function $B *$ by

$$
\mathbf{x}-\mathbf{B}^{*}(\mathbf{x})=\Pi(\mathbf{x}) \text {. }
$$

From (9) and (10),

$$
B^{*} \cdot(x)=\left[x-B^{*}(x)\right]\left[\lambda+\frac{f_{Y}(x \mid x)}{F_{Y}(x \mid x)}\right] .
$$

Now choose functions $g_{Y}(x \mid x)$ and $G_{Y}(x \mid x)$ to satisfy

$$
\frac{g_{Y}(x \mid x)}{G_{Y}(x \mid x)}=\left[\lambda+\frac{f_{Y}(x \mid x)}{F_{Y}(x \mid x)}\right] .
$$


Hence $g_{Y}(x \mid x) /\left[G_{Y}(x \mid x)\right]$ satisfies Lemma 1 of Milgrom and Weber [12, p. 1107]. Hence the ficitious bidding function $B^{*}$ satisfies the hypotheses of Theorem 16 of Milgrom and Weber [12, p. 1109]; in part.icular, B* satisfies the Milgrom Weber equation (7). Applying this result, we conclude that EB* is nondecreasing with the release of affiliated information, and so, from (10), EI is nonincreasing with information release. Q.E.D. For the special case of risk-neutral bidders, $B^{*}=B$. Thus the seller cannot make himself worse off by revealing information in the first-price sealed-bid auction. This is Theorem 16 of Milgrom and Weber [12, p. 1109]. However, only in the case of risk-neutral bidders is it necessarily the case that releasing information cannot lower the expected revenue in the first-price sealed-bid auction. With risk-averse bidders, the expected revenue can fall. To see this, denote by $B(x, s)$ the equilibrium bid when the seller reveals affiliated information $s$, and by $B(x)$ the bid when the seller conceals the information. Then for risk-averse bidders $(\lambda>0)$, Theorem 1 implies

$$
E[\exp \{\lambda B(x, s)]] 1_{\{Y \leq X]} \geq E[\exp [\lambda B(x)\}] 1_{\{Y \leq X\}}{ }^{\prime}
$$

where the last term on each side is the indicator function. This does not necessarily imply that $\mathrm{EB}(x, s) \geq \mathrm{EB}(x)$, so it does not necessarily imply more expected revenue for the seller. We conclude from (13) that, in a first-price sealed-bid auction with risk-averse bidders, the seller may either increase or decrease his expected revenue by revealing affiliated information.

Consider now the more special independent-private-values model. With valuations drawn independently, $F_{Y}(y \mid x)=F_{Y}(y)$, and (4) becomes 


$$
\frac{f_{Y}(x \mid x)}{F_{Y}(x \mid x)}=\frac{d}{d x} \log F_{Y}(x)
$$

Thus (7) becomes

$$
\begin{aligned}
U(B(x), x) & =F_{Y}(x) \int_{0}^{X} e^{-\lambda(x-\alpha)} e^{-\log \left(F_{Y}(x)-F_{Y}(\alpha)\right)} d \alpha \\
& =\int_{0}^{X} e^{\lambda(\alpha-x)} F_{Y}(\alpha) d \alpha .
\end{aligned}
$$

What are the effects of the seller's releasing some affiliated information $s$ in the independent-private-values model? Denote by $F_{Y}(X, s)$ the distribution of the first-order statistic of the others' values when the bidder knows the information $s$. Note that the distribution when he does not know $s, F_{Y}(x)$, is equal to $E_{S} F_{Y}(x, s)$, where $E_{S}$ is expectation with respect to s.

THEOREM 2. In a first-price sealed-bid auction in which bidders have independent private values and constant absolute risk aversion, any bidder with given valuation is equally well off whether the seller has a policy of revealing or concealing affiliated information.

Proof. The ex ante expected utility of a bidder with valuation $x$ under a policy of announcement is, from (15),

$$
\begin{aligned}
\operatorname{ES}_{\mathbf{S}} U(B(x, s), x) & =E \int_{S}^{x} e^{\lambda(\alpha-x)} F_{Y}(\alpha, s) d \alpha \\
& =\int_{0}^{x} e^{\lambda(\alpha-x)} E_{S} F_{Y}(\alpha, s) d \alpha \\
& =\int_{0}^{x} e^{\lambda(\alpha-x)} F_{Y}(\alpha) d \alpha \\
& =\operatorname{EU}(B(x), x) .
\end{aligned}
$$$$
\text { Q.E.D. }
$$ 
The foregoing result compared the policies of concealment and revelation from the point of view of a bidder who knows his own valuation. There is another notion of ex ante, namely, the situation before bidders know their own valuations. Theorem 2 implies that

$$
\int_{0}^{\bar{x}} E U(B(x, s), x) \operatorname{Prob}\left(X_{1}=x\right) d x=\int_{0}^{\bar{x}} U(B(x), x) \operatorname{Prob}\left(x_{1}=x\right) d x
$$

Thus bidder 1 , before he draws his valuation $x$, is indifferent between the two policies.

Which policy will the seller choose?

THEOREM 3. In a first-price sealed-bid auction with independent private values, concealing information does not decrease the seller's revenue.

Proof. From Theorem 2,

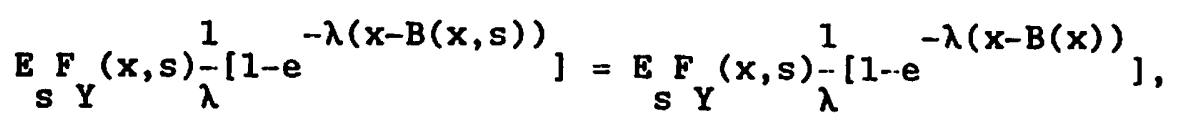

from which

$$
\operatorname{EF}_{S}(x, s) e^{\lambda[B(x, s)-B(x)]}=E_{S Y}(x, s),
$$

or

$$
E_{s}\left[\frac{F_{Y}(x, s) e^{\lambda[B(x, s)-B(x)]}}{E_{S} F(x, s)}\right]=1 .
$$

Thus, by convexity,

$$
e^{E s^{\lambda[B(x, s)-B(x)]}} \leq E_{s}\left[\frac{F Y(x, s) e^{\lambda[B(x, s)-B(x)]}}{E_{S Y Y}^{F}(x, s)}\right]=1,
$$

which implies $\mathrm{E}_{s} \lambda[B(x, s)-B(x)] \leq 0$, with strict inequality if $F_{y}$ depends on $s$ and $\lambda \neq 0$. Q.E.D. 
Equality holds in (20) if and only if either the bidders are risk neutral (that is, $\lambda=0$ ), or the information does not alter the probability distribution over the highest of the other bidders' valuations (that is, $F_{Y}(x, s)$ does not depend on $\left.s_{.}\right)$Apart from these two special cases, the policy of concealment results in strictly higher revenues than the policy of revelation.

Milgrom and Weber [12] obtained the following results. With risk-neutral bidders, the release of information by the seller raises the expected selling price in a first-price sealed-bid, Vickrey, or English auction. With risk-averse bidders releasing information raises the price.in an English or a Vickrey auction. The results obtained above show that, in a first-price sealed-bid auction with risk-averse bidders (the case not examined by Milgrom and Weber), information release can result in either an increase or a decrease in the expected price.

The tendency for the selling price to rise after information release when bidders' valuations are affiliated is caused by what Milgrom and Weber [12] and Milgrom [10] called the linkage effect. A rough intuition for this is as follows (for a more precise description, see Milgrom [10]). It is intuitively clear that, in general, any reduction in the variance of the bidders' estimates of the item's value increases bidding competition and drives up the price. The release of information links bidders' estimates to the public information: it reduces the advantages from private information. In other words, the release of information has a similar effect to a reduction in the variance of perceived valuations.

Theorems 1 and 3 above show that, with risk-averse bidders in a first-price sealed-bid auction, there is, in addition, a contrary tendency for the release of information to drive down the price. To understand this 
effect, consider the case when it exists in isolation of the linkage effect, that is, when bidders' values are independent. In this case, a policy of revealing information would not on average change bidding behavior in the Vickrey or English auction: the expected second-highest valuation remains unchanged. The bidders may be better off, however, because, with constant absolute risk aversion, revealing information reduces the risk premium (Milgrom and Weber [12, Theorem 20, p. 1115]). In contrast, in a first-price sealed-bid auction with risk-averse bidders, revealing information does change bidding behavior. If the revealed information is good news then each bidder knows that his rivals will bid more aggressively, so he must do likewise. Bad news similarly generates less aggressive bidding. Thus the policy of revealing information results in a higher variance of bids than the policy of concealing information. It therefore results in a lower price on average. We shall call this effect the bid-dispersion effect.

Milgrom [10] pointed out that linkages increase the randomness in bidders' payoffs. The foregoing results are consistent with this. From Theorem 2, bidders with independent private values in a first-price sealed-bid auction are indifferent between the policy of revealing information and the policy of concealing information. From Theorem 3, bids are on average lower with information revelation. It follows that the risk-averse bidders must be faced with more risk under revelation than under concealment.

Thus the bid-dispersion effect is absent from a Vickrey or English auction or when bidders are risk neutral; it operates by itself in a first-price sealed-bid auction with risk-averse bidders having independent valuations; and it operates counter to the linkage effect in a first-price sealed-bid auction with risk-averse bidders having affiliated valuations. 
The usual examples of information that can be revealed by the seller (an expert's appraisal of a painting, a geological survey of an oil well, etc.) do not apply to the independent-private-values model, since each bidder is assumed to know the value of the item to him. There is, however, one type of information that is useful to a bidder when valuations are independent: information about the amount of competition.

Suppose there is a finite set of potential bidders $A$. The set of active bidders is drawn from $*$, and the number of bidders is statistically independent of the bidders' valuations. Preserve symetry by supposing that all active bidders perceive the same probability distribution over the number of active bidders. It is clear that information about the number of active bidders is at least weakly affiliated, so that the hypotheses of the model are satisfied by this particular type of information. Moreover, the distribution of the highest of the other bidders' valuations, $F_{Y}(x, s)$, clearly depends nontrivially on $s$ when $s$ represents information about the number of active bidders. Hence, provided $\lambda>0$, condition (20) holds with strict inequality. Thus, in a first-price sealed-bid auction with independent private values and strictly risk-averse bidders (having constant absolute risk aversion), the seller's expected revenue is strictly higher when he conceals the number of bidders than when he reveals it. 5

In an extension of the present analysis, Matthews [6] has shown that the result that the seller prefers to conceal the number of bidders continues to hold when the bidders have decreasing absolute risk aversion. However, the bidders prefer a policy of revelation when they have decreasing absolute rjsk aversion, and a policy of concealment when they have increasing absolute risk aversion. 
In an English auction, the price is the same whether or not the number of bidders is known, since in either case the bidding stops at the second-highest valuation. In the independent-private-values model with risk-averse bidders, the English auction and the Vickrey auction yield lower revenue for the seller than the first-price sealed-bid auction with the number of bidders known (Riley and Samuelson [15]); in turn, as we have seen, this is dominated by the first-price sealed-bid auction with an unannounced number of bidders. Thus, with risk-averse bidders, the auction form considered above, the first-price sealed-bid auction with the number of bidders concealed, is the best of the simple auction forms from the point of view of the seller. 


\section{The Optimal Auction with a Stochastic set of Bidders}

What is the optimal auction when the number of bidders is unknown? For the sake of tractability, we restrict attention now to the case of risk-neutral bidders. 6

Suppose there is a finite set of potential bidders $k=\{1,2, \ldots, \bar{n}\}$. Let $\beta_{A}$ denote the probability that $A \subseteq *$ is the set of active bidders. The bidders have Bayesian-consistent prior probabilities over the set of active bidders.

The process by which potential bidders become active bidders (that is, the determination of the probabilities $\beta_{A}$ ) is regarded as exogenous. For example, in the case of government-contract bidding with particular firms invited to submit bids, the government chooses the bidders on a rotating basis from a list of qualified firms (McAfee and McMillan [9, Ch. 8]). Drop last section's assumption that potential bidders are identical. Suppose instead that potential bidder $i$ independently draws his valuation $x_{i}$ from a distribution $F_{i}$, which may vary from bidder to bidder; assume $F_{i}(0)=0$ and let $F_{i}^{\prime}=f_{i}$. Thus informing bidders about the number of active bidders is now not the only issue; bidders also may or may not be informed about the identities of the other active bidders. The perceived probability of any potential bidder actually bidding may depend upon his valuation distribution $F_{i}$. (For example, it may be that a potential bidder who values the good 
highly is more likely actually to submit a bid than a potential bidder with a low valuation. Thus it is possible that, if $F_{1}(x)<F_{2}(x)$ for all $x$, then $\beta_{\{1\} \cup_{A}} \geq \beta_{\{2\} \cup A}$ for all $\left.A, 1,2 \notin A.\right)$ Although valuations are drawn independently from the $F_{i} \cdot s$, this formulation admits some perceived correlations among valuations. (For example, suppose the set of bidders is $\{1,2,3,4,5\} ;(\forall x) F_{1}(x)=F_{2}(x) \geq F_{3}(x)=F_{4}(x) ; \beta_{\{1,2,5\}}=1 / 2$, $\beta_{\{3,4,5\}}=1 / 2, \beta_{A}=0$ for all other $A$. Then it would appear to potential bidder 5 that the other bidders' valuations were correlated.)

The problem just defined is the optimal-auction problem solved by Myerson [14], but generalized in one respect: in this problem the bidders know neither how many other active bidders there are, nor the identities (that is, the $F_{i}$ 's) of the other active bidders. Myerson's case is obtained in the analysis to follow by setting $\beta_{A}=1$ and $\beta_{B}=0$ for $B \neq A$, where $A$ represents Myerson's known set of bidders.

We simplify the analysis relative to Myerson's by assuming the distributions $F_{i}$ satisfy the following regularity condition:

(21) $\frac{d}{d x}\left(x-\frac{1-F_{i}(x)}{f_{i}(x)}\right)>0$.

(Compare with Myerson [14, p. 66].) This simplifies the analysis because, when (31) fails, the seller must randomize (Maskin and Riley [2]).

Suppose the seller values the object at $x_{0} \geq 0$. The seller uses an incentive-compatible direct mechanism by announcing sets $\Gamma_{i}^{A} \subseteq R|A|, i \in A$, and functions $\alpha_{i}^{A}: \quad{ }^{|A|} \rightarrow R$ so that, if the set of actual bidders is $A$ and the bidders report valuations $x^{A}$, then each bidder $i \in A$ pays an amount $\alpha_{i}^{A}\left(x^{A}\right)$ and is awarded the good if $x^{A} \in \Gamma_{i}^{A}$. 
We use the following notation:

(22) $\left\{a_{1}, \ldots, a_{|A|}\right\}=A$;

(23) $\left(x_{-a}^{A}, z\right)=\left(x_{a}, \ldots, x_{a_{i-1}}, z, x_{i+1}, \ldots, x_{|A|}\right)$;

(24) $\Gamma_{0}^{A}=R^{|A|} \backslash\left(\underset{i \in A}{U} \Gamma_{i}^{A}\right)$;

(25) $\underset{-a_{i}}{d x^{A}}=d x_{1} \ldots d a_{i-1}{ }_{a} a_{i+1} \cdots d a_{|A|}$;

(26) $f^{A}\left(x^{A}\right)=\operatorname{mifA}_{i}\left(x_{i}\right)$;

(27) $\Delta_{i}^{A}(z)=\left\{x_{-i}^{A} \mid\left(x_{-i}^{A}, z\right) \in \Gamma_{i}^{A}\right\}$, if $i \in A$;

(28) $\mu_{i}^{A}(z)=\int_{\Delta_{i}^{A}(z)} \prod_{\substack{j \in A \\ j \neq i}} f_{j}\left(x_{j}\right) d x_{-i}^{A}$, if $i \in A$;

(29) $\sigma_{i}^{A}(z)=\int_{R} \underset{|A|-1}{ } \alpha_{i}^{A}\left(x_{-i}^{A}, z\right) \prod_{\substack{j \neq i \\ j \in A}} f_{j}\left(x_{j}\right) d x_{-i}^{A}$.

Here (24) defines the set of reports $x$ for which the seller keeps the good. If the $x^{\prime} s$ represent true evaluations, (23) gives the vector of responses when bidder $i$ reports $z$ and everyone else is honest. (27) gives the set of others' valuations for which $i$ wins the good with a report of $z$. (28) gives the probability that $i$ wins with the report $z$, while (29) gives i's expected payment with the report $z$. Let $\sum_{w}$ denote the sum over $w$ satisfying $R(w)$. 
The incentive-compatibility constraints are

(30) $\underset{i \in A}{\sum_{A} \beta_{A}\left[z \mu_{i}^{A}(z)-\sigma_{i}^{A}(z)\right]}$

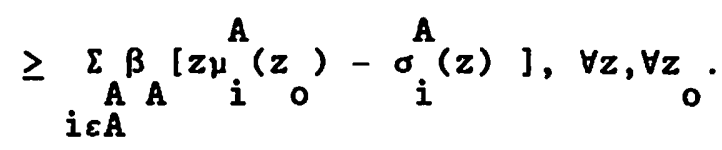

The free-exit constraints are

$$
\left.\underset{i \in A}{\sum_{A}^{A} \beta_{A}[z \mu}{ }_{i}^{A}(z)-\sigma_{i}^{A}(z)\right] \geq 0, \forall z .
$$

THEOREM 4. The seller maximizes his expected revenue by setting $\Gamma_{1}^{A}$ to satisfy:

(32) $\Gamma_{i}^{A}=\left\{x \in R^{|A|} \mid x_{i}-\frac{1-F_{i}\left(x_{i}\right)}{f_{i}\left(x_{i}\right)} \geq \max \left[x_{0}, x_{j}-\frac{1-F_{j}\left(x_{j}\right)}{f_{j}\left(x_{j}\right)}\right]\right\}$.

Proof. Rewrite the incentive-compatibility constraints:

$$
\sum_{i \in A}^{A} \beta_{A}\left[\sigma_{i}^{A}(z)-\sigma_{i}^{A}(z)\right] \leq \sum_{i \in A}^{A} \beta_{A} z\left[\mu_{i}^{A}(z)-\mu_{i}^{A}(z)\right]
$$

and

(34) $\sum_{i \in A} \beta_{A}\left[\sigma_{i}^{A}(z)-\sigma_{i}^{A}(z)\right] \geq \underset{i \in A}{\sum_{A} \beta_{A} z\left[\mu_{i}^{A}(z)-\mu_{i}^{A}(z)\right] 0}$.

Divide by $z-z$ and take limits to yield

(35) $\sum_{i \in A}^{A} \beta_{A}\left[\sigma_{i}^{A}(z)\right]=\sum_{i \in A}^{A} A_{A} z \mu_{i}^{A}(z)$,

should these derivatives exist. The free exit constraint (31) implies $\sigma_{i}^{A}(0) \leq 0$; but the seller wants $\sigma_{i}^{A}(0)$ to be as large as possible, so that 
$\sigma_{i}^{A}(0)=0$. Hence

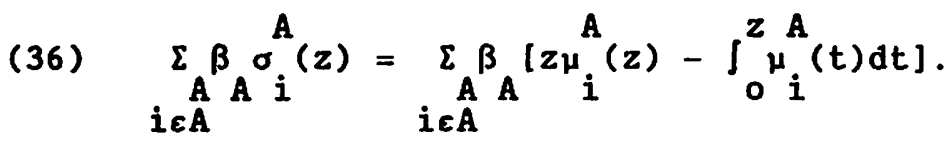

The seller expects to earn:

(37) $\Phi=\sum_{A A^{\beta}}\left\{x_{0} \int_{\Gamma_{0}^{A}} f^{A}\left(x^{A}\right) d x^{A}+\sum_{i \in A} \int_{R}|A|^{\alpha}{ }_{i}^{A}\left(x^{A}\right) f^{A}\left(x^{A}\right) d x^{A}\right\}$

$=\sum_{A A_{0} \beta}\left[x\left[1-\sum_{i \in A} \Gamma_{i}^{A} \Gamma_{i}^{A}\left(x^{A}\right) d x^{A}\right]+\sum_{i \in A} \int_{0}^{\infty} \sigma_{i}^{A}(z) f_{i}^{A}(z) d z\right\}$

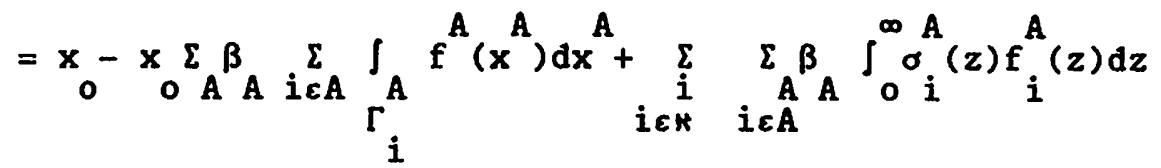

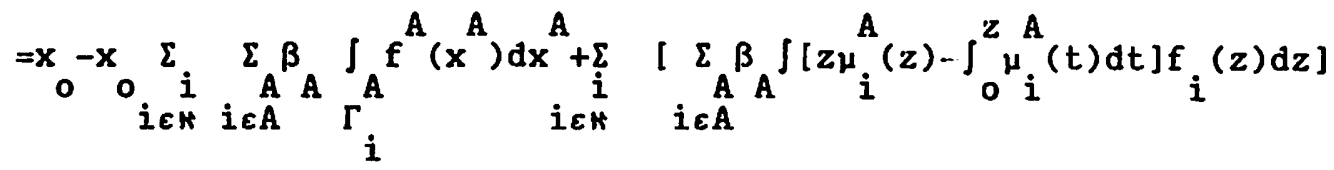

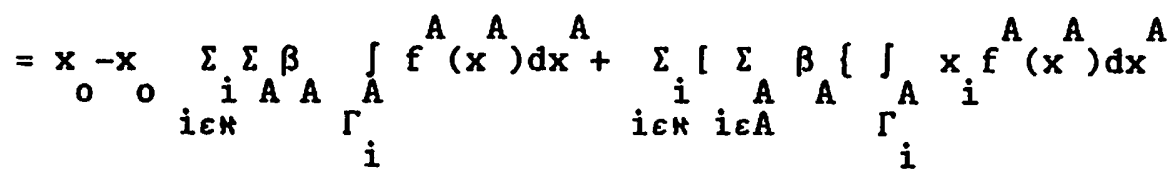

$\left.\left.+\left[\left[1-F_{i}(z)\right] \int_{0}^{z} \mu_{i}^{A}(t) d t\right]{ }_{0}^{\infty}-\int_{0}^{\infty}\left[1-F_{i}(z)\right] \mu_{i}^{A}(z) d z\right]\right]$

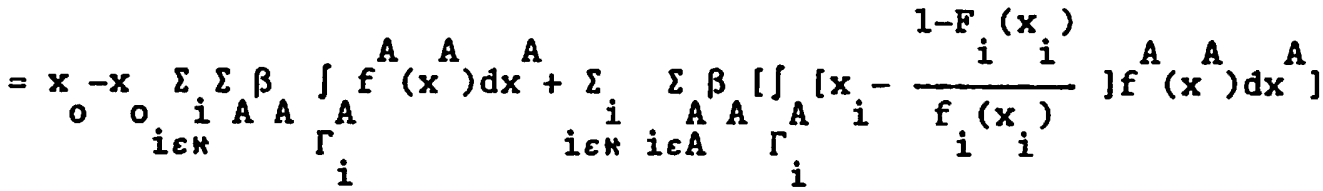

$=x_{0}+\sum_{i \in A}^{i} \sum_{A C_{A}^{A}}^{\beta}\left[\int_{\Gamma_{i}^{A}}\left[x_{i}-x_{0}-\frac{1-F_{i}\left(x_{i}\right)}{f_{i}\left(x_{i}\right)}\right] f^{A}\left(x^{A}\right) d x^{A}\right]$ 


$$
=x_{0}+\sum_{A \subseteq A}^{\beta}{ }_{A} \sum_{i \in A} \int_{\Gamma_{i}}\left\{x_{i}-x_{0}-\frac{1-F_{i}\left(x_{i}\right)}{f_{i}\left(x_{i}\right)}\right\} f^{A}\left(x^{A}\right) d x
$$

Maximizing $\Phi$ with respect to $\Gamma_{i}^{A}$, subject to $\Gamma_{i}^{A} \cap \Gamma_{j}^{A}=\Phi$ for $i \neq j$, $i, j \in$ A yields, from Stokes' Theorem (Sagan [14, p. 542]),

(38) $\Gamma_{i}^{A}=\left\{x_{A} \mid x_{i}-x_{0}-\frac{1-F_{i}\left(x_{i}\right)}{f_{i}\left(x_{i}\right)} \geq \max \left[0, x_{j}-x_{0}-\frac{1-F_{j}\left(x_{j}\right)}{f_{j}\left(x_{j}\right)}\right]\right\}$.

Condition (38) gives the unconstrained maximizer of $\Phi$. However, $\Phi$ must be maximized subject to the constraint that the function $\Sigma_{A} \beta_{A} \mu_{i}^{A}(z)$ is nondecreasing in $z$ (which is necessary for incentive compatibility). The regularity condition (21) ensures that the unconstrained maximizer in (38) does in fact satisfy the monotonicity constraint.

Q.E.D.

Since the payment function $\sigma_{i}^{A}$ is contingent on the set of bidders $A$, the seller implicitly reveals the set of bidders when he announces the payment function. However, if the seller wishes to conceal the set of bidders, he can make payment not contingent on A (provided he knows the probabilities $\beta_{A}$ ) by using the payment function

(40)

$$
\sigma_{i}(z)=\frac{\sum_{A}{ }_{A} \sigma_{i}^{A}(z)}{\sum_{i \in A}{ }_{A} A} .
$$

The noteworthy feature of Theorem 4 is that the optimal auction does not depend on the probabilities over the sets of active bidders, $\beta_{A}$. Thus the optimal auction derived by Myerson [14] when the set of bidders is common knowledge remains the optimal auction when the bidders do not know the set of bidders. This implies the following result. 
COROLLARY. The seller's maximum expected revenue with risk-neutral bidders having independent private values is the same whether or not the bidders know the set of bidders.

Consider the case in which $F_{i}=F_{j}=F$ for all $i, j \in k$ : all bidders draw their valuations from the same distribution. Then Theorem 4 says that the seller optimally sets a reserve price $r$ satisfying $x_{0}=r-(1-F(r)) / f(r)$ and chooses the highest remaining bidder (as in Myerson [14] and Riley and Samuelson [15]). If $F_{i} \neq F_{j}$ for some $i, j \in *$, then reserve prices are again used but the optimal auction discriminates against certain bidders, in that a lower-valuation bidder can win the item despite the presence of a higher-valuation bidder. (See Myerson [14] and McAfee and McMillan [8] for details.)

How can the optimal auction be implemented in practice in the case of symmetric bidders (that is, $F_{i}=F_{j}$ for all $i, j \varepsilon *$ )? When the set of bidders is common knowledge, it is well known that the optimal direct, incentive-compatible auction can be mimicked by either an English auction or a first-price sealed-bid auction, provided appropriate reserve prices are imposed (Milgrom [10], Myerson [14], Riley and Samuelson [15]). Does this remain the case when the bidders do not know the set of bidders? suppose that the bidders' expectations over the set of bidders, while Bayesian consistent, are not identical. Then the optimal auction can be implemented using an English auction, because in the English auction, the second-last bidder drops out of the bidding when the bids reach the value of the second order statistic; this gives the seller the same expected revenue as the optimal direct auction of Theorem 4. However, it is important to note that the optimal auction cannot be implemented by a first-price sealed-bid 
auction when bidders have different expectations. This is because the bidding functions of different bidders will fail to coincide; as a result, it is possible that the highest bidder in the first-price sealed-bid auction is not the appropriate winner as defined by the optimal $\Gamma_{i}^{A}$. Note also that the converse of this applies: if the bidders have different expectations over the set of bidders, then only for a measure-zero set of $F_{i}{ }^{\prime} s$ is the first-price sealed-bid auction optimal. Thus we have a result contrary to the Revenue-Equivalence Theorem, even though bidders are symmetric, risk neutral, and have independent private values.

\section{Conclusion}

The results of auction theory are sensitive to the assumption that the set of bidders is common knowledge. In a first-price sealed-bid auction with bidders who have independent private values and are risk averse (with constant absolute risk aversion), the expected selling price is strictly higher when the bidders do not know how many other bidders there are than when they do know this. In an ex ante sense, any bidder is indifferent between the policy of being told and the policy of not being told the number of bidders. With risk-neutral bidders, the optimal auction is the same whether or not the bidders know who their competitors are. However, this optimal auction may not be implementable using a first-price sealed-bid auction, although it is implementable using an English auction.

More generally, in a first-price sealed-bid auction with bidders who are risk averse and have affiliated private values, the release of any affiliated information by the seller generates two opposing tendencies: the linkage effect, identified by Milgrom and Weber [12], which tends to raise the selling price; and the bid-dispersion effect identified above, which tends to lower the selling price. 
The limits of the foregoing results should be stressed. As Milgrom and Weber [12] pointed out, the independent-private--values assumption (used in part of Section 2 and in section 3 ) is restrictive: it requires that each bidder has no doubt about the value of the item to him, and that there be no possibility of reselling the item later at some as yet unknown price. The analysis of section 2 assumed constant absolute risk aversion: Matthews [6] showed that some of the results change when this assumption is relaxed. The results of section 3 depend upon the assumption of risk neutrality, as is shown by the results on optimal auctions with risk-averse bidders of Maskin and Riley [3] and Matthews [4]. 


\section{Footnotes}

*We thank an Associate Editor and a referee for their perceptive comments, and the Ontario Economic Council for research support.

${ }^{1}$ See Milgrom $[10,11]$ for surveys of auction theory.

2 For example, Ontario Hydro, Ontario's electrical utility, has a policy of keeping secret the number of firms it has invited to submit bids: see HcAfee and McMillan [9, Ch. 8].

${ }^{3}$ A more detailed comparison between the analysis and results of Milgrom and Weber [12] and this paper will be given in Section 2 .

4 Note that equation (6) is the risk-aversion analogue of equation (7) of Milgrom and Weber [12, p. 1107]. This is the point at which the assumption of constant absolute risk aversion is used. The differential equation (6) defining the bidding function $B$ is a linear differential equation if and only if the bidders have constant absolute risk aversion (McAfee and McMillan [7]).

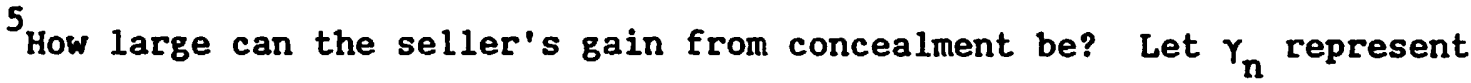
the objective probability that there are $n$ active bidders. In a simulation with $\gamma_{2}=\gamma, \gamma_{1}=1-\gamma, \lambda=1$ and $F$ uniform on $[0,1]$, concealment increases revenue by $25 \%$ for small $\gamma$, and by $10 \%$ for $\gamma=1 / 3$. In addition, the percentage increase in revenue is a decreasing function of $\gamma$ in this simulation.

${ }^{6}$ On optimal auction design with risk-averse bidders and a fixed, known set of bidders, see Maskin and Riley [3], Matthews [4,5], and Moore [13].

7 Note that bidders can have differing expectations over the set of bidders even when $F_{i}=F_{j}$ for all $i, j \in *$. For example, let there be two potential bidders: potential bidder 1 always bids, while potential bidder 2 bids with probability $\alpha<1$. Let $p_{n}^{i}$ denote bidder i's probability that the number of active bidders is $n$ conditioned on he himself being an active bidder. Then $p_{1}^{1}=1-\alpha \neq 0=p_{1}^{2}$. 


\section{References}

1. Ralph Cassady, Jr., "Auctions and Auctioneering," University of California Press, Berkeley, 1967.

2. Eric Maskin and John Riley, Monopoly with incomplete information, Working Paper No. 268, UCLA, August 1983.

3. Eric Maskin and John Rj ley, Optimal auctions with risk averse buyers, Econometrica 52 (1984): 1473-1518.

4. Steven A. Matthews, Selling to risk averse buyers with unobservable tastes, Journal of Economic Theory 30 (1983): 370-400.

5. Steven A. Matthews, On the implementabjlity of reduced form auctions Econometrica 52 (1984): 1519-1522.

6. Steven A. Matthews, On comparing auctions for risk averse buyers: a buyer's point of view, Discussion Paper No. 664, Northwestern University, September 1985.

7. R. Preston McAfee and John McMillan, Bidding for contracts: A principalagent analysis, mimeo, University of Western Ontario, 1984.

8. R. Preston McAfee and John McMillan, Discrimination in auctions, Discussion Paper 85-7, University of California, San Diego, January 1985.

9. R. Preston McAfee and John McMjllan, "Incentives in Government Contracting," (Toronto: Ontario Economic Council, 1985), to be published.

10. Paul Milgrom, Auction theory, presented to the World Congress of the Econometric Society, 1985.

11. Paul Milgrom, The economics of competitive bidding: a selective survey, in "Social Goals and Social Organization," L. Hurwicz, D. Schmeidler and H. Sonnenschein (eds.) Cambridge: Cambridge University Press, 1985 .

12. Paul Milgrom and Robert $J$. Weber, $A$ theory of auctions and competitive bidding, Econometrica 50 (1982): 1089-1122.

13. John Moore, Global incentive constraints in auction design, Econometrica 52 (1984): 1523-1536.

14. Roger B. Myerson, Optimal auction design, Mathemathics of operations Research 6 (1981): 58-73.

15. John Riley and William Samuelson, Optimal auctions, American Economlc Review 71 (1981): 381-392.

16. H. Sagan, "Advanced Calculus," Houghton Mifflin, Boston, 1974. 